\section{WS THE WEB OF EBM RECOMMENDATIONS}

${ }^{1} \mathrm{M}$ Ploegmakers, ${ }^{2} \mathrm{~T}$ van Vegchel, ${ }^{1} \mathrm{M}$ Molag, ${ }^{2} \mathrm{M}$ Tilma, ${ }^{2} \mathrm{~S}$ Kersten, ${ }^{1} \mathrm{~T}$ van Barneveld. ${ }^{1}$ Knowledge Institute of Medical Specialists, Utrecht, Netherlands; ${ }^{2}$ Comprehensive Cancer Centre of the Netherlands (IKNL), Utrecht, Netherlands

\section{0:1136/bmjgs-2013-002293.30}

Background Guidelines on specific diseases and interventions are connected to other guidelines on more general topics (eg perioperative care) or to other specific guidelines on comorbidity. Together all recommendations form a web of EBM knowledge. When searching for a recommendation for a specific type of patient, illness and comorbidity, it should be found immediately, without turning many pages. Furthermore, information should be unambiguous, correct and up to date. Therefore, in the Netherlands a web-based database was developed that contains all available CPG's, cut into ready-to-use modules. Each module easily shows connections with related content like other modules, background information, flowcharts, summaries and implementation tools. Moreover, the web-based presentation facilitates updating of modules, without revising the whole $\mathrm{CPG}$, making this process more flexible and efficient.

Objectives - To present the modular set-up including the connections between related content of the Dutch Guideline Database. - To discuss how modular CPG-presentation can bring EBM closer to clinical practice and facilitate updating.

Target Group This workshop will be of most interest for clinicians and guideline developers interested in optimising EBM dissemination, efficient updating and implementation.

Description - Illustrate the need for a modular database based on examples from guidelines on anaesthesia. - Discuss quality regulation of content. - Discuss the role of patient involvement in the database. - Discuss to what extent content elements should be related to justify a 'built-in' relation (complexity of the web of recommendations). - Discuss possible connexions between the Dutch Guideline Database and the G-I-N Database.
073WS COLLABORATIVE GUIDELINE IMPLEMENTABILITY TOOL DEVELOPMENT AND EVALUATION

${ }^{1} \mathrm{~J}$ Cheng, ${ }^{1} \mathrm{~A}$ Gagliardi, ${ }^{2} \mathrm{~B}$ Melissa, ${ }^{3} \mathrm{O}$ Bhattacharyya. ${ }^{1}$ University Health Network; ${ }^{2}$ Department of Oncology, McMaster University, Hamilton, Canada; ${ }^{3} \mathrm{Li}$ Ka Shing Knowledge Institute of St. Michael's Hospital, Toronto, Canada

\section{0:1136/bmjqs-2013-002293.31}

Background Research shows that guidelines are more easily translated to practice when accompanied by information that helps users accommodate, implement and evaluate use of the recommendations. We refer to this information in guidelines or other resources as guideline implementability tools (GItools). We identified exemplar GItools, generated criteria by which to assess and develop GItools through consultation with G-I-N members, and created an online directory for sharing of GItools. Discussion with G-I-N members would help us improve these resources and identify partners for collaborative GItool development. This work is a core activity of GIRAnet: the Guideline Implementability Research and Application Network (www.g-i-n.net/activities/ implementation/giranet).

Objectives To share information about GItool resources; gather feedback on how to improve GItool resources; learn about other GItool initiatives; and establish partners for joint development or evaluation of GItools. AUDIENCE Guideline developers, implementers, users (clinicians, managers, policy-makers) and researchers. Description A brief presentation will define GItools and describe their potential purpose and impact; review methods used to identify, describe and evaluate exemplar GItools; and demonstrate the GItool Directory (20 minutes). Participants will be asked to individually rate, then discuss enhancements for Gitool assessment criteria (20 $\mathrm{min})$. Open discussion will enable sharing and mutual learning about other GItool initiatives, and explore partnerships for future GItool initiatives (40 min). Remaining time will be used to identify priorities for ongoing research (10 min). Participant feedback will be used to improve GItool resources, and guide ongoing GIRAnet projects. 